\title{
Demographic Profile of Hearing Deficiency in a Peripheral Referral Hospital - A Five Year Study
}

Amit Chakrabarti, ${ }^{1}$ Indranil Sen, ${ }^{1}$ Rupam Sinha, ${ }^{1}$ Manish Kumar, ${ }^{1}$ Rabi Hembrom, ${ }^{1}$ Satadal Mandal, ${ }^{1}$ Amit Bikram Maiti ${ }^{1}$

\author{
Introduction \\ This present study is an attempt to study the demographic characteristics of individuals presenting with hearing loss in a \\ peripheral tertiary care hospital using the available database of pure tone audiometry results. \\ Materials and Methods \\ A retrospective, observational, descriptive study was conducted over a period of 5 years in the Department of ENT of a peripheral \\ tertiary care hospital wherein all subjects having a pure tone audiometry were included in the study. The records of PTA done \\ were analyzed followed by systematic analysis. \\ $\underline{\text { Results }}$ \\ A total of 9790 individuals inclusive of 5566 males and 4224 females were studied. Maximum number of individuals belonged \\ to the age group of 21-30 years. Nearly $63 \%$ of total subjects suffered from Bilateral hearing loss. Sensorineural hearing loss \\ was most prevalent type of loss. Mild degree of loss was most prevalent. Adolescents and young adults comprised the bulk of the \\ study population. Sensorineural hearing is the most common type. Bilateral hearing loss is more frequent. Mild degree of loss \\ is most frequent.

\section{Conclusion} \\ This study emphasized the 'Composite parameter distribution' in addition to the classical single parameter demographic \\ profile of deafness in a remote tertiary care hospital. Adolescents and young adults comprised the bulk of the study population. \\ Sensorineural hearing is the most common type. Bilateral hearing loss is more frequent. Mild degree of loss is most frequent. \\ $\underline{\text { Keywords }}$ \\ Audiometry, Pure Tone; Hearing Loss; Demography
}

$\underline{\text { ABSTRACT }}$

$\mathrm{T}$ The ear is a marvellously complex and sensitive organ. Unfortunately, damage to the organ, whether through disease, physical insult, long term exposure to excessive noise, some drugs or simply the effects of aging, can cause the ear to malfunction. The result of malfunction is usually to produce some degree of deafness. ${ }^{1}$

Diseases of the ear have profound effect on the health and quality of life of millions of people around the globe. A significant proportion of cases of hearing loss are due to common ear diseases i.e. Ear wax, External auditory canal infections, Otomycosis, ASOM, CSOM, OME etc, which if diagnosed early and managed properly can significantly reduce the burden of decreased hearing. '(Table I)

According to Census 2011 data of India; nearly 1 in every 5 differently capable individuals suffers from hearing loss.

The ability to communicate is a crucial aspect of human life as auditory sense is very important for communication of any kind. Hearing impairment is a major morbidity which is often undiagnosed and more often unreported in peripheral healthcare centres of our country. For proper feedback and planning regarding future management, a proper database is mandatory which is often lacking in our system. Thus this present study is an attempt to study the demographic characteristics of individuals presenting with hearing loss in a peripheral tertiary care hospital

1 - Department of ENT, Midnapore Medical College,

Midnapore

Corresponding author:

Dr Rupam Sinha

email: rupamsinhaad2@gmail.com 
Table I: Disease load of hearing loss estimated by WHO (2012) ${ }^{2}$

7328 million (91\%) of these are adults (183 million males, 145 million females) and 32 (9\%) million of these are children

\section{The prevalence of disabling hearing loss in children is greatest in South Asia, Asia Pacific and Sub-} Saharan Africa.

Approximately one-third of persons over 65 years are affected by disabling hearing loss.

The prevalence of disabling hearing loss in adults over 65 years is highest in South Asia, Asia Pacific and Sub-Saharan Africa.

using the available database of pure tone audiometry results.

\section{Materials and Methods}

The study was conducted in the Department of otorhinolaryngology of a peripheral tertiary care hospital over a period of five years (2012-16). The study design was retrospective, observational and descriptive in nature. Patients presenting with chief complaint of hearing loss, after clinical examination, were subjected to Pure tone audiometry. The records of pure tone audiometry done during the study period underwent systematic analysis following predesigned pretested semi-structured protocol. ${ }^{3}$ The study excluded children below 5 years of age, individuals with severe mental or physical disabilities and the individuals clinically established to be malingering. The data was analyzed to study the demographic characteristics of individuals based on different parameters e.g. age, sex, type, extent and severity of hearing loss among the study population. (Refer to Proforma in Annexure 1 and 2)

\section{Results}

A total of 9790 individuals inclusive of 5566 males $(56.85 \%)$ and 4224 females $(43.14 \%)$ had a pure tone audiogram done during the study period in our institute and were included in the study after screening. Maximum number of individuals belonged to the age group of 21-30 years $(n=1946 ; 19.87 \%)$ followed by $11-20$ years $(\mathrm{n}=1838 ; 18.77 \%)$. (Table II). Altogether a total of 579 individuals were excluded during screening as per exclusion criteria mentioned above.

The distribution of study population based on age showed similar results between both sexes with 1130 males $(20.30 \%)$ falling in $21-30$ years age group followed by 1002 males (18\%) belonging to $11-20$ years age group. In females 836 individuals (19.79\%) belonged to $11-20$ years age group followed by 816

Table II: Table showing distribution of study population based on age

\begin{tabular}{|c|c|c|}
\hline AGE GROUPS & TOTAL & PERCENTAGE \\
\hline $06-$ Oct & 813 & 8.3 \\
\hline Nov-20 & 1838 & 18.77 \\
\hline $21-30$ & 1946 & 19.87 \\
\hline $31-40$ & 1506 & 15.38 \\
\hline $41-50$ & 1559 & 15.92 \\
\hline $51-60$ & 1140 & 11.64 \\
\hline $61-70$ & 703 & 7.18 \\
\hline $71-80$ & 236 & 2.41 \\
\hline$>=81$ & 49 & 0.5 \\
\hline GRAND TOTAL & 9790 & 100 (approx) \\
\hline
\end{tabular}




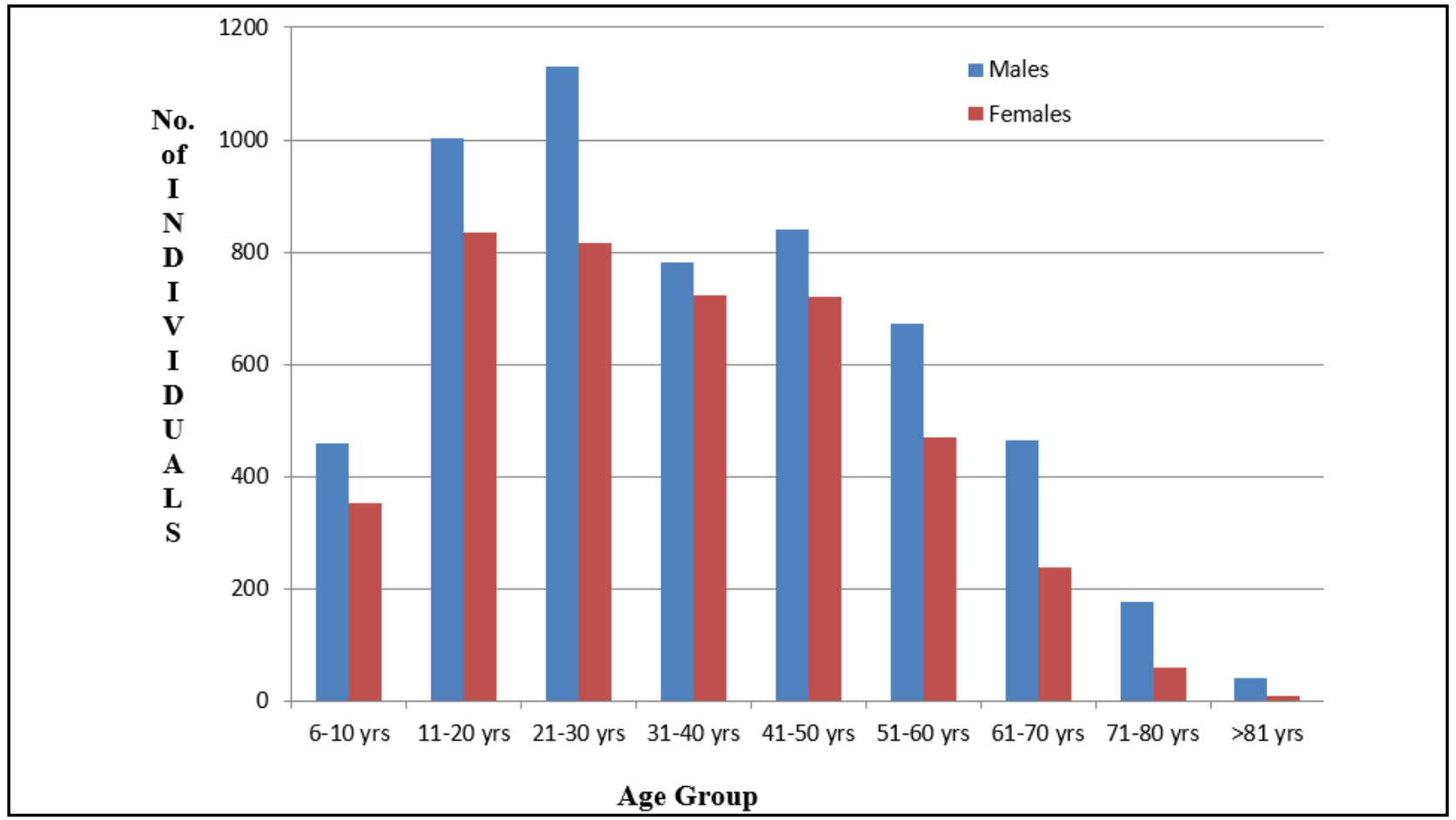

Fig. 1. Clustered bar diagram showing distribution of gender according to age groups

individuals (19.31\%) in 21-30 years age group. (Fig-1)

Among 9790 individuals, 6157 (62.89\%) suffered from bilateral hearing loss [3494 males $(62.77 \%)$ and 2663 females (63.04\%)].

As far the 'Nature of deafness and the laterality is concerned, 4522 individuals (46.19\%) had sensorineural hearing deficit in right ear and 4468 individuals (45.64\%) in left ear. Conductive deafness was present in 1543 individuals $(15.76 \%)$ in right ear and 1735 individuals $(17.72 \%)$ in left ear. Mixed deafness was present in 778 individuals (7.94\%) in right ear and 811 individuals $(8.28 \%)$ in left ear. (NOTE: Remainder of the persons had normal hearing for that ear)

As far as the 'Gender distribution of the deafness' is concerned, $48.79 \%$ Males had SNHL in right ear and $48.02 \%$ in left ear and conductive hearing loss was present in $12.57 \%$ individuals in right ear and $14.39 \%$ subjects in left ear. Mixed deafness was seen in 6.91\% cases in right ear in $7.74 \%$ cases of left ear. Amongst females; $42.75 \%$ individuals had SNHL in right ear and $42.49 \%$ in left ear. Conductive hearing loss was present in $19.96 \%$ individuals in right ear and $22.11 \%$ subjects in left ear. Mixed deafness was seen in $8.36 \%$ cases in right ear in $8.99 \%$ cases of left ear.

The 'Age distribution of Deafness' was as followsConductive deafness was greatest amongst individuals of $11-20$ years $(4.00 \%$ in right ear and $4.42 \%$ in left ear). Sensori-neural loss was highest in $41-50$ years age group in right ear (7.68\%) and in left ear (7.55\%) was seen 51-60 years age group. Mixed loss has been found to be greatest in $41-50$ years age group $(1.77 \%$ in right ear and $1.93 \%$ in left ear).

The 'Degree of hearing loss', as per classification forwarded by Waleed et $\mathrm{al}^{4}{ }^{4}$, it was noted as- of the 9790 individuals tested, 2605 (26.6\%) individuals had mild hearing loss in right ear and $2716(27.74 \%)$ in left ear. Moderate loss was seen in 1705 (17.41\%) in right ear and $1682(17.18 \%)$ in left ear. Moderately severe hearing deficit manifested in 1039 cases $(10.61 \%)$ of right ear and 1071 cases $(10.93 \%)$ of left ear. Severe degree of loss was seen in $968(9.88 \%)$ of right ear and $971(9.91 \%)$ of left ear. Profound deafness was found in $527(5.38 \%)$ of right ear and $549(5.60 \%)$ cases of left ear. Remainder of the individuals had normal hearing. 
Table III: Degree of hearing loss in relation to gender

\begin{tabular}{|c|c|c|c|c|c|}
\hline $\begin{array}{c}\text { GENDER \& } \\
\text { SIDE }\end{array}$ & $\begin{array}{c}\text { MILD HL } \\
(25-40 \text { DB) }\end{array}$ & $\begin{array}{c}\text { MODERATE } \\
\text { HL (41-55 } \\
\text { DB) }\end{array}$ & $\begin{array}{c}\text { MODERATELY } \\
\text { SEVERE HL (56- } \\
70 \text { DB) }\end{array}$ & $\begin{array}{c}\text { SEVERE HL (71- } \\
90 \text { DB) }\end{array}$ & $\begin{array}{c}\text { PROFOUND HL } \\
\text { (>90 DB) }\end{array}$ \\
\hline Males (Right) & $25.29 \%$ & $16.33 \%$ & $10.68 \%$ & $10.27 \%$ & $5.76 \%$ \\
\hline Males (Left) & $27.25 \%$ & $15.97 \%$ & $10.90 \%$ & $10.47 \%$ & $5.64 \%$ \\
\hline Females (Right) & $28.33 \%$ & $18.84 \%$ & $10.51 \%$ & $9.37 \%$ & $4.87 \%$ \\
\hline Females (Left) & $28.38 \%$ & $18.77 \%$ & $10.98 \%$ & $9.18 \%$ & $5.56 \%$ \\
\hline
\end{tabular}

Degree of hearing loss showed similar results across both sexes. (Table III)

\section{Discussion}

The disease burden estimations based on sound epidemiological research provide the foundation for appropriate public policy focus and measures for effective management of disease conditions. A significant proportion of cases of hearing impairment (HI) are due to common ear diseases, which if diagnosed early and managed properly can significantly reduce the burden of decreased hearing.

In the present study maximum number of individuals belonged to the age group of 21-30 years followed by 11-20 years and 31-40 yrs. Guleria et $\mathrm{a}^{1}$ conducted a community based cross sectional study in an urban area where it was found that $29.4 \%$ of individuals belonged to the age group of 31-45 years. This correlates well with the age distribution in the present study and also to the fact that the results of this present hospital based study, an institute which caters to both adjacent rural and urban population, can be extrapolated to the general population. ${ }^{1}$ With increasing life expectancy along with changes in diet and lifestyle; exposure to chronic noise, ototoxic chemicals and drugs is on the rise with consequent rise in old age deafness with up to $40 \%$ individuals above 75 years suffering from disabling hearing loss. ${ }^{5}$ Deafness due to old age has been found to be up to eight times more common in USA. ${ }^{6}$
Among 9790 individuals in the present study, 6157 $(62.89 \%)$ suffered from bilateral hearing loss which comprised of 3494 males $(62.77 \%)$ and 2663 females (63.04\%). In the study conducted by Guleria et al, $60 \%$ of the male population was found to be suffering from hearing loss. ${ }^{1}$ Prevalence of hearing loss in males was found to be greater in the study conducted by Kalpana et al. ${ }^{7}$ Even in USA, the prevalence of hearing impairment predominantly affected males. ${ }^{6}$ As far as laterality of hearing impairment is concerned, the study conducted by Guleria et al shows no difference between right and left sides but bilateral impairment was more frequently encountered than unilateral hearing loss. ${ }^{1}$ In the study conducted by Asghari et al, the prevalence of hearing impairment had no significant association with gender. ${ }^{8}$

Sensori-neural hearing loss was predominant in the present study and also in the study conducted by Guleria et $\mathrm{al},{ }^{1}$ whereas conductive pathology dominated in the study by Kalpana et al. ${ }^{7}$ This difference can be attributed to the fact that the study by Kalpana et al involved only school going children rather than general population. Mixed deafness affected least number of individuals in all the articles reviewed. In the present study conductive deafness was greatest amongst individuals of 11-20 years; sensori-neural loss was highest in 41-50 years age group. Mixed loss has been found to be greatest in 41-50 years age group.

In the present study $(26.8 \%)$ individuals had mild hearing loss in right ear and $(27.74 \%)$ in left ear. Moderate loss was seen in (17.6\%) in right ear and 
$(17.37 \%)$ in left ear. Moderately severe hearing deficit manifested in $(10.6 \%)$ of right ear and $(10.93 \%)$ of left ear. Severe degree of loss was seen in $(9.82 \%)$ of right ear and $(9.82 \%)$ of left ear. Profound deafness was found in $(5.31 \%)$ of right ear and $(5.60 \%)$ cases of left ear. (Note: The results are the average of both sexes). These findings are in unison with the study conducted by Guleria et al wherein mild to moderate degree of hearing loss predominated across the study population. ${ }^{1}$ Similar findings were noted amongst the Korean population in the study conducted by Hong et al. ${ }^{9}$ The study conducted by Goman et al in USA shows highest prevalence of mild degree of hearing impairment which is in concordance with our study. ${ }^{10}$ Even the study conducted by Asghari et al on Iranian population revealed most number of individuals with grade 1 hearing impairment with an increase in grade of hearing loss with increasing age. ${ }^{8}$

\section{Conclusion}

The demographic profile of the deafness population is sine qua non for any comprehensive planning to address the problem. However, profile of individual parameter e.g. age, gender, type of deafness etc. often does not reflect the actual burden of the problem. In that case, distribution of composite parameters e.g. 'Age related type of deafness' or 'Age related degree of deafness' become more helpful. In the present study, effort has been made to emphasise this aspect of 'Composite parameter distribution' in addition to the classical single parameter demographic profile of deafness in a remote tertiary care hospital.

\section{References}

1. Guleria TC, Mohindroo S, Mohindroo NK, Azad RK. Prevalence and etiology of hearing impairment in urban area of Shimla, Himachal Pradesh, India: a cross sectional observational study. Int J Res Med Sci. 2017 Apr;5(4):1252-5

2. Mortality and burden of diseases and prevention of Blindness and Deafness WHO, 2012. WHO global estimates on prevalence of hearing loss

3. Wilson, David H., 1942- \& Centre for Population Studies in Epidemiology (S.A.) 1998, Hearing impairment in an Australian population, Centre for Population Studies in Epidemiology, Dept. of Human Services, [Adelaide]
4. Waleed B. Alshuaib, Jasem M. Al-Kandari and Sonia M. Hasan (December 2nd 2015). Classification of Hearing Loss, Update On Hearing Loss, Fayez Bahmad Jr., IntechOpen, DOI: 10.5772/61835. Available from: https://www.intechopen.com/ books/update-on-hearing-loss/classification-of-hearing-loss

5. Varshney S. Deafness in India. Indian J Otol. 2016; 22:73-6

6. Holt J, Hotto S, Cole K. Demographic aspects of hearing impairment: Questions and answers. Third Edition. Washington, DC: Gallaudet University; 1994

7. Kalpana R, Chamyal PC. Study of prevalence and aetiology of the hearing loss amongst school going children. Indian Journal of Otolaryngology and Head \& Neck Surgery. 1997; 49(2):1424

8. Ashgari A, Farhadi M, Daneshi A, et al. The Prevalence of Hearing Impairment by Age and Gender in a Population-based Study. Iranian Journal of Public Health 2017; 46(9):1237-46

9. Hong JW, Jeon JH, Ku CR, Noh JH, Yoo HJ, Kim DJ. The prevalence and factors associated with hearing impairment in the Korean adults: the 2010-2012 Korea National Health and Nutrition Examination Survey (observational study). Medicine 2015; 3;94(10): e611

10. Goman AM, Lin FR. Prevalence of Hearing Loss by Severity in the United States. Am J Public Health 2016 Oct; 106(10):1820-

\section{Acknowledgment}

The authors acknowledge the contribution of the Audiometry assistant, MMC\&H. Mrs. Namita Samanta for taking the pains of maintaining the Audiometry Data for the last few decades in order. 


\section{ANNEXURE 1}

Proforma of Recording the Audiological Evaluation used in our study

\section{Name:}

2. Age:

3. Sex:

4. Type of Hearing Loss a. Right ear: Conductive/Sensori-neural/mixed

b. Left ear:Conductive/Sensori-neural/mixed

5. Degree of Hearing Loss

a. Right ear: Mild/Moderate/Moderately severe/ Severe/Profound

b. Left ear: Mild/Moderate/Moderately severe/ Severe/Profound

6. Unilateral/Bilateral Hearing Loss

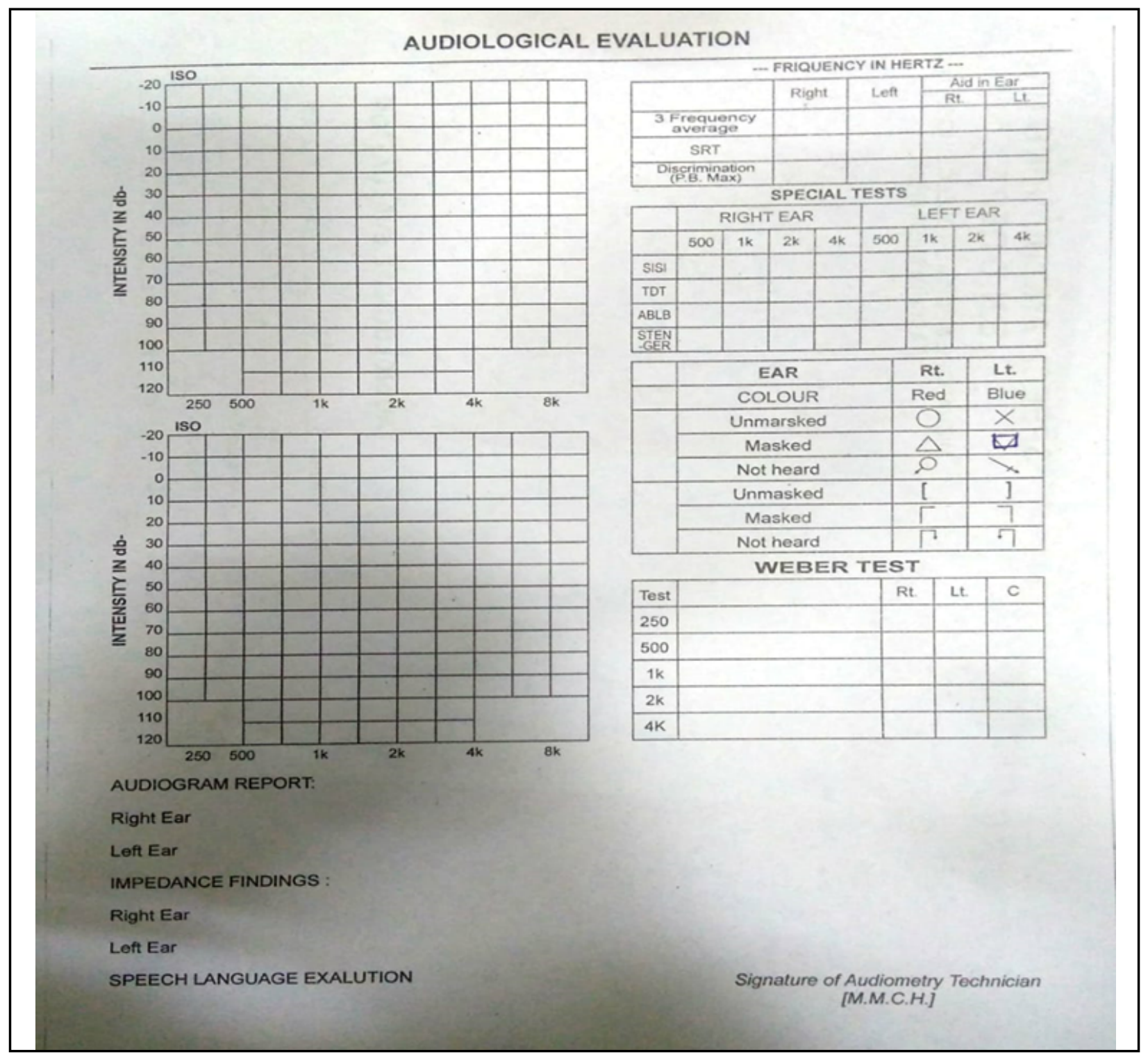


ANNEXURE 2

(Proforma used in Reference Number 3)

SOUTH AUSTRALIAN HEARING STUDY

Location :

File No:

Name:

Date of Birth

Main Lifetime Occupation:

Sex: M/F

This audiogram shows:

Left Right

[ ] [ ] Normal hearing across all frequencies

[ ] [ ] Sensorineural hearing loss

[ ] [ ] Conductive hearing loss

Overall degree

[ L ] Mild/Moderate/Severe/Profound

[ R ] Mild/Moderate/Severe/Profound

Unilateral/Bilateral

Left Right

[ ] [ ] Better ear

[ ] [ ] Worse ear

[ ] Symmetrical

Date:

Tester.

Recommendations

- Monitor hearing levels regularly

- Wear hearing protection

- Seek further audiological services

- Consider hearing aid fitting

- Contact G.P. for ENT investigation

- Consider hearing support services eg Better hearing Aust

- Other

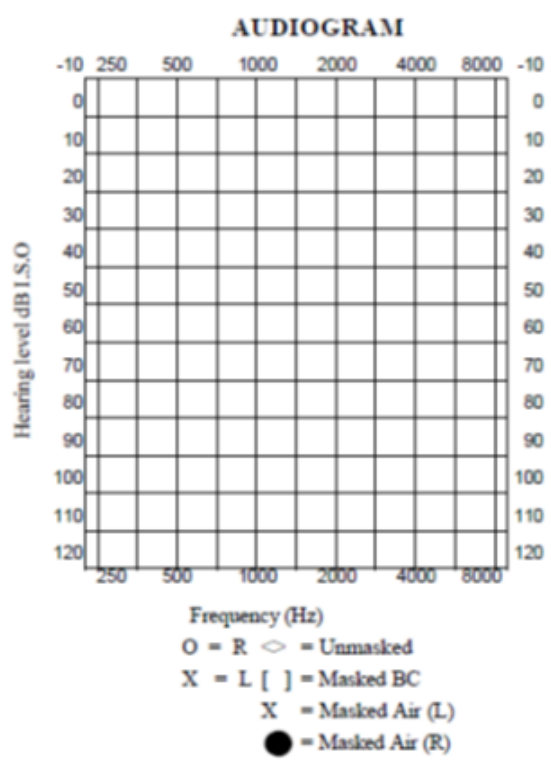

Hearing Threshhold Levels

\begin{tabular}{|r|l|l|l|l|l|l|l|l|}
\hline Ear & 250 & 500 & 1000 & 2000 & 3000 & 4000 & 6000 & 8000 \\
\hline Left air & & & & & & & & \\
\hline bone & - & & & & - & & - & - \\
\hline Right air & & & & & & & & \\
\hline bone & - & & & & - & & - & - \\
\hline
\end{tabular}

The South Australian Hearing Study (a National Health \& Medical Research Council research project) acknowledges the support of the AUDIOLOGICAL SOCIETY OF AUSTRALIA INC.

Audiologists are University Graduates who have extensive specialist training at Post-graduate level.

Audiologists specialise in the assessment, prevention and non-medical management of hearing impairment and associated disorders of communication 